\title{
ANALYSIS OF POWER SAVING CLASS II TRAFFIC IN IEEE 802.16E WITH MULTIPLE SLEEP STATE AND BALKING
}

\author{
Chinmaya Misra * Veena Goswami ${ }^{\dagger}$
}

\begin{abstract}
The battery life of Mobile Stations in IEEE 802.16e can be extended substantially by applying the sleep mode mechanism. This paper studies an efficient method to analyze the performance of the power saving class type II for delay sensitive traffic in multiple sleep state. The incoming data frames may join or balk the buffer due to impatience with some probability. We present an $M / M / 1 / N$ queueing model with balking and multiple vacations in order to exhibit the self-similar property of IEEE 802.16e. We develop a cost function to determine the optimal service rate that minimizes the total expected cost. Various performance indices such as the average number of data frames in the system, the mean waiting time of the data frame in the system, the the average balking rate due to impatience, etc. have been presented. Numerical results are provided to show the influence of various parameters on the behavior of the system. The proposed model provides a tradeoff between the average abandon rate and the power consumption.
\end{abstract}

Keywords: IEEE 802.16e, power saving class type II, balking, queueing, performance indices, sleep state.

\section{Introduction}

WiMAX is an emerging broadband wireless technology that fulfills the promise of considerably widening the reach of broadband internet services, with the additional benefit that it gets rid of huge deployment costs. Recently developed IEEE 802.16e systems offer scalability in both radio access technology and network architecture,

\footnotetext{
*School of Computer Application, KIIT University, Bhubaneswar - 751024, India, Email:cmisra@yahoo.com

${ }^{\dagger}$ School of Computer Application, KIIT University, Bhubaneswar - 751024, India, Email: veena_goswami@yahoo.com
} 
thus rendering a great deal of flexibility in network deployment choices and service offerings to the Mobile Stations (MS). An additional operation like handover is included in the IEEE 802.16e standard to offer user mobility. This supports seamless handoff between Base Stations (BS) and Mobile Stations in vehicular speeds without interrupting the connection as depicted in Figure 1. The MS are fully dependent on lightweight batteries for maintaining the connections with the serving Base Stations. The IEEE 802.16 standard family adopt power saving mechanism by discontinuing connection for a pre-negotiated period of time with the BS.

To render high-quality services for mobile stations, various sleep mode procedures have been suggested to reduce the power consumption of MS with restricted battery capacity. The sleep mode operation in IEEE 802.16e standard proposes three types of power saving classes: I, II and III for different service types [3, 6]. Among the three types, power saving class type I is suitable for best-effort and non-real-time variable rate (NRT-VR) services. The dynamic sleep cycle length and fixed listening interval are considered in power saving class type I. Power saving class type II is suitable for the connections of unsolicited grant service and real-time variable rate (RT-VR) services. In this case, fixed/dynamic listening interval and fixed sleep cycle length are considered for enhancing the power saving. During listening windows of power saving class type II the MS may send or receive any service data units without interruption of sleep mode. Thus, power saving class type II have the potential of avoiding unnecessary switching cost. Power saving class type III is suitable for multicast connections as well as for management operations.

IEEE 802.16e sets a sleep mode operation for maintaining the power of mobile terminals to support battery powered mobile wireless broadband access systems efficiently. Sleep mode is proposed to minimize MS power usage and decrease usage of air interface resources within serving BS. In the sleep mode, the MS is allowed a series of alternate listening windows and sleep windows. The listening window is a time period in which the MS is required to transmit/receive data in the same way as in state of normal operation. During the sleep window, the BS shall not transmit data or management messages to the MS. The mobile WiMAX supports strict QoS for real-time streaming multimedia applications that guarantee a certain level of performance to a data flow, like required bit rate, delay, jitter or packet dropping probability [16]. But real time streaming applications of power saving class type II require low end-to-end delay to maintain streaming nature of the traffic [8]. On the other side, BS buffers the service data units destined to the MS for a predetermined period. If the delay is more than the maximum tolerable threshold, the data frames are eventually dropped at the MAC layer after reaching MS. This causes extra overhead and wastage of energy. So, there is a tradeoff between the power saving and the delay of the data traffic.

The downlink traffic in power saving class type II of IEEE 802.16e have been studied extensively in $[3,7,15]$. An analytical model and analysis of the specified sleep mode in terms of energy consumption and packet delay was considered in [18]. A brief summary of work addressing energy efficient and low-power design for wireless network protocol has been reported in Jones et al. [11]. Lee et al. [12] discussed power saving schemes for a VoIP connection in the context of IEEE 802.16e standard. They developed an algorithm to find the length of the sleep interval based on the 


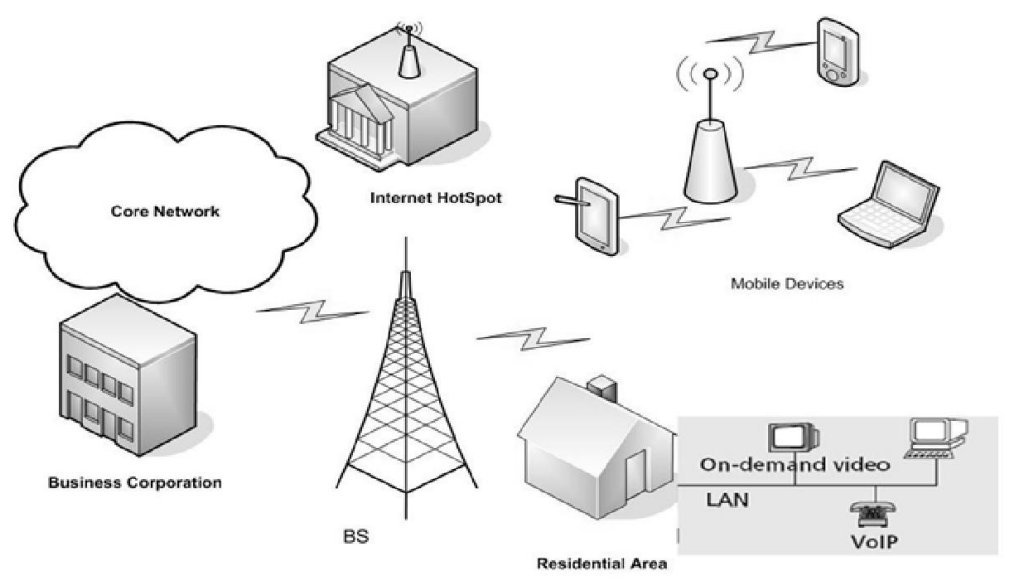

Figure 1: Infrastructure of IEEE 802.16

network delay model for end-to-end VoIP connection. Yue et al. [17] studied a finite buffer Markovian queueing system with balking, reneging and server vacations. Baek and Choi [1] presented a scheme that ensures minimum length for effective power saving, by considering the benefits of sleep modes in both the IEEE 802.16e and IEEE $802.16 \mathrm{~m}$. The impact of both downlink and uplink traffic and an analytical model for the sleep mode operation of the IEEE $802.16 \mathrm{~m}$ has been reported in [2]. The power saving mechanism with binary exponential traffic indications has been presented in Hwang et al. [4]. Hwang et al. [5] attempts to reduce the state transition overhead between the awake and sleep modes, and thus, it enhances the power-saving in the IEEE 802.16e standard. An enhanced power saving class of type III in the IEEE 802.16e network has been studied in [9]. Jin et al. [10] conducted an analytical study on the power consumption in the IEEE 802.16 standard by extending the listening window and adjusting the sleep cycle length. Power saving mechanisms that activate both type I and type II power saving classes have been presented in [13]. The energy consumption reduces by increasing the unavailability interval.

The multiple sleeps in power saving scheme with balking accommodate the real time streaming data more closely in real-life situations. In practice, mobile users become impatient when they do not receive their desired data. Moreover, the delay sensitive real life applications discard their data frames due to excess delay after reaching the MS. This motivated us to study and apply an $M / M / 1 / N$ queueing system with balking and multiple sleeps in order to implement energy saving scheme efficiently. The main issue is to maximize power saving, while maintaining the required QoS on end-to-end delay and the packet loss for real time streaming data of power saving class type II. Very few analytical attempts have considered the impatience of data frames in mobile WiMAX. In the proposed paper, impatience probability has been derived based on number of frames waiting in the BS buffer.

The rest of the paper is organized as follows. Section 2 presents the description of the system model and its analysis. Performance indices of the system model have 
been discussed in Section 3. Based on the performance indices, we formulate the total expected cost function to determine the optimal service rate at minimum cost using quadratic fit search method. The performance measures such as the average number of data frames in the system, the mean waiting time of the data frame in the system, the the average balking rate due to impatience, etc. are also elaborated upon in the section. In Section 4, some numerical results are presented to establish the various parameters influence on the behavior of the system. Section 5 concludes the paper.

\section{System Model}

The energy consumption and data frame delay are two key components in the design of an efficient power saving mechanism. The system model is developed for the data frames of power saving class type II (Real time traffic) in IEEE 802.16e. When the MS has no data frame to transmit or receive during an intended period, it sends $M O B-S L P-R E Q$ message to the BS requesting to transit into sleep mode through the sleep controller. It then begins to settle into the sleep mode after receiving the $M O B-S L P-R S P$ response message returned by the BS. The MS state list present in BS maintains a record of each MS's present sleep state. After the MS gets into sleep mode, the BS determines $M O B-T R F-I N D$ to negative intimation and changes state list at the same time. If a data frame intended to the MS arrives at the BS during this period of time, the data frame will be buffered and the $M O B-T R F-I N D$ is set to positive intimation. If the message $M O B-T R F-I N D$ indicates a positive intimation, the BS changes the MS state to awake-mode and sends the data frames normally. The sleep mode operation in power saving class type II initiated by the BS is illustrated in Figure 2.

Let us consider the finite buffer fed by the downlink data frames at the the base station. The data frames of power saving class type II arrive at the buffer according to Poisson process with arrival rate $\lambda$. In WiMAX, the transmission of data frames supports point to multiple mode (PMP), that is, data can be transmitted from one BS to multiple MS. But here we consider the downlink data frames from BS to individual MS. The service time is assumed to be exponentially distributed with mean $1 / \mu$. If MS server is idle, then the data frame on arrival at BS Buffer, will be serviced immediately. Let $b_{n}$ represent the probability with which the incoming data frames decide to join the buffer and let $1-b_{n}$ be the probability that they decide to balk, when there are $n$ incoming data frames ahead of them in the system. Further, we assume $0 \leq b_{n+1} \leq b_{n}<1,1 \leq n \leq N-1, b_{0}=1$ and $b_{N}=0$. Here the sleep period is assumed to be exponentially distributed with mean $1 / \phi$. The traffic intensity is given by $\rho=\lambda / \mu$. The data frames are served on a first-come first-served (FCFS) buffer discipline. The inter-arrival times, sleep periods and service times during normal busy period are mutually independent.

Let us assume at steady-state $P_{n, 0}, 0 \leq n \leq N$, be the probability that there are $n$ data frames in the system when server is in sleep mode, and $P_{n, 1}, 1 \leq n \leq N$, be the probability that there are $n$ data frames in the system when server is in awake 
BS

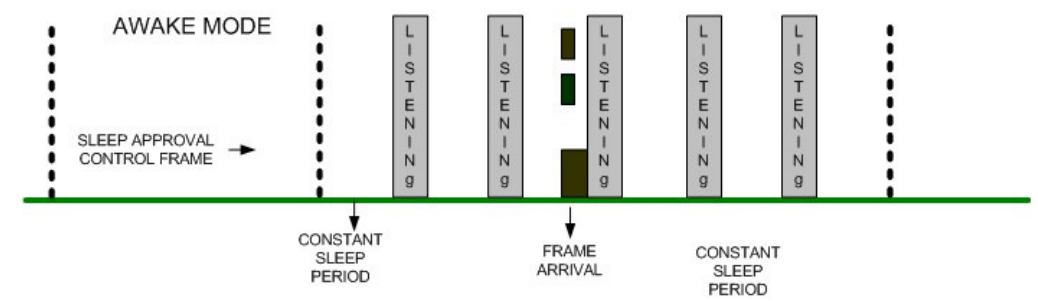

Figure 2: Sleep operation of the power saving class type II initiated by the BS.

mode. Using the Markov process, we get the following equations:

$$
\begin{aligned}
\lambda P_{0,0} & =\mu P_{1,1} \\
\left(\lambda b_{n}+\phi\right) P_{n, 0} & =\lambda b_{n-1} P_{n-1,0}, 1 \leq n \leq N-1, \\
\phi P_{N, 0} & =\lambda b_{N-1} P_{N-1,0} \\
\left(\lambda b_{1}+\mu\right) P_{1,1} & =\phi P_{10}+\mu P_{2,1}, \\
\left(\lambda b_{n}+\mu\right) P_{n, 1} & =\lambda b_{n-1} P_{n-1,1}+\phi P_{n, 0}+\mu P_{n+1,1}, 1 \leq n \leq N-1, \\
\mu P_{N, 1} & =\lambda b_{N-1} P_{N-1,1}+\phi P_{N, 0} .
\end{aligned}
$$

Now, the stationary probabilities $P_{n, 0}$ and $P_{n, 1}$ can be derived by using equations (1) - (5) in terms of $P_{N, 0}$. Solving recursively (2) and (3), we obtain

$$
P_{n, 0}=\psi_{n} P_{N, 0}, \quad 0 \leq n \leq N,
$$

where $\psi_{N}=1, \psi_{N-1}=\frac{\phi}{\lambda b_{N-1}}$ and

$$
\psi_{n}=\frac{\phi}{\lambda b_{N-1}} \prod_{j=n}^{N-2}\left(\frac{\lambda b_{j+1}+\phi}{\lambda b_{j}}\right), 0 \leq n \leq N-2 .
$$

From (1), (4) and (5), we get

$$
P_{n, 1}=\xi_{n} P_{N, 0}, \quad 1 \leq n \leq N,
$$

where $\xi_{1}=\frac{\lambda}{\mu}, \quad \xi_{2}=\left(\frac{\lambda b_{1}}{\mu}+1\right) \xi_{1}-\frac{\phi}{\mu} \psi_{1}$ and

$$
\xi_{n}=\frac{1}{\mu}\left[\left(\lambda b_{n-1}+\mu\right) \xi_{n-1}-\lambda b_{n-2} \xi_{n-2}-\phi \psi_{n-1}\right], \quad 3 \leq n \leq N .
$$

Using normalization condition $\sum_{n=0}^{N} P_{n, 0}+\sum_{n=1}^{N} P_{n, 1}=1$, we get

$$
P_{N, 0}=\left[1+\sum_{n=0}^{N-1} \psi_{n}+\sum_{n=1}^{N} \xi_{n}\right]^{-1} .
$$




\section{$3 \quad$ Performance indices}

In this section, we discuss some performance indices of the proposed system. The probability that the system is in a awake mode $(P(B))$ and the probability that the system is in a sleep mode $(P(V))$ can be obtained as

$$
P(B)=\sum_{n=1}^{N} P_{n, 1}, \quad P(V)=\sum_{n=0}^{N} P_{n, 0}=1-P(B) .
$$

The average number of data frames in the system $\left(L_{s}\right)$ and the average number of data frames in the buffer $\left(L_{q}\right)$ are given by

$$
\begin{aligned}
L_{s} & =\sum_{n=1}^{N} n\left(P_{n, 0}+P_{n, 1}\right), \\
L_{q} & =\sum_{n=0}^{N} n P_{n, 0}+\sum_{n=1}^{N}(n-1) P_{n, 1} .
\end{aligned}
$$

Using Little's rule, we obtain the mean waiting time of the data frame in the system $\left(W_{s}\right)$ and the mean waiting time of the data frame in the buffer $\left(W_{q}\right)$, respectively, as

$$
W_{s}=\frac{L_{s}}{\lambda^{\prime}} \quad \text { and } \quad W_{q}=L_{q} / \lambda^{\prime}
$$

where data frame loss due to buffer size limitation is $P_{l o s s}=P_{N, 0}+P_{N, 1}$ and effective data frame arrival rate is $\lambda^{\prime}=\lambda\left(1-P_{\text {loss }}\right)$.

As the probability that an incoming data frame balks or drops from the system is $1-b_{n}$, the instantaneous balking rate is $\lambda\left(1-b_{n}\right)$. Thus, we can obtain the average balking rate $(B R)$ as

$$
B R=\sum_{n=1}^{N} \lambda\left(1-b_{n}\right) P_{n, 0}+\sum_{n=1}^{N} \lambda\left(1-b_{n}\right) P_{n, 1} .
$$

When there are $n$ data frames in the system, the average rate of joining the system (JS) is given by

$$
J S=\sum_{n=1}^{N} \lambda b_{n} P_{n, 0}+\sum_{n=1}^{N} \lambda b_{n} P_{n, 1}
$$

\section{Cost function}

We develop the expected cost function per slot for the $M / M / 1 / N$ queue with balking and multiple vacations. Our aim is to determine optimal service rate $\mu$, say $\mu^{*}$ in awake mode. By taking into consideration the performance system characteristics, 
we build a cost function $F(\mu)$ to minimize the expected cost per slot. Let us assume the following cost factors:

$C_{1}=$ Cost incurred per slot for keeping the MS in awake mode.

$C_{2}=$ Reward of power preservation per slot when the MS is in sleep mode.

$C_{3}=$ Cost of the average number of data frames in the BS buffer.

$C_{4}=$ Cost due to balking of data frames from the BS buffer.

Using the definitions of cost factors listed above and its corresponding performance system indices, we obtain the expected operating cost per slot as

$$
F(\mu)=C_{1} P(B)+C_{2} P(V)+C_{3} L_{q}+C_{4} B R .
$$

The computation of derivatives of the expected cost function (9) is very complex. Therefore, the computation of the optimum value of $\mu$ is not straightforward. We apply the quadratic fit search method to solve the optimization problem. We assume

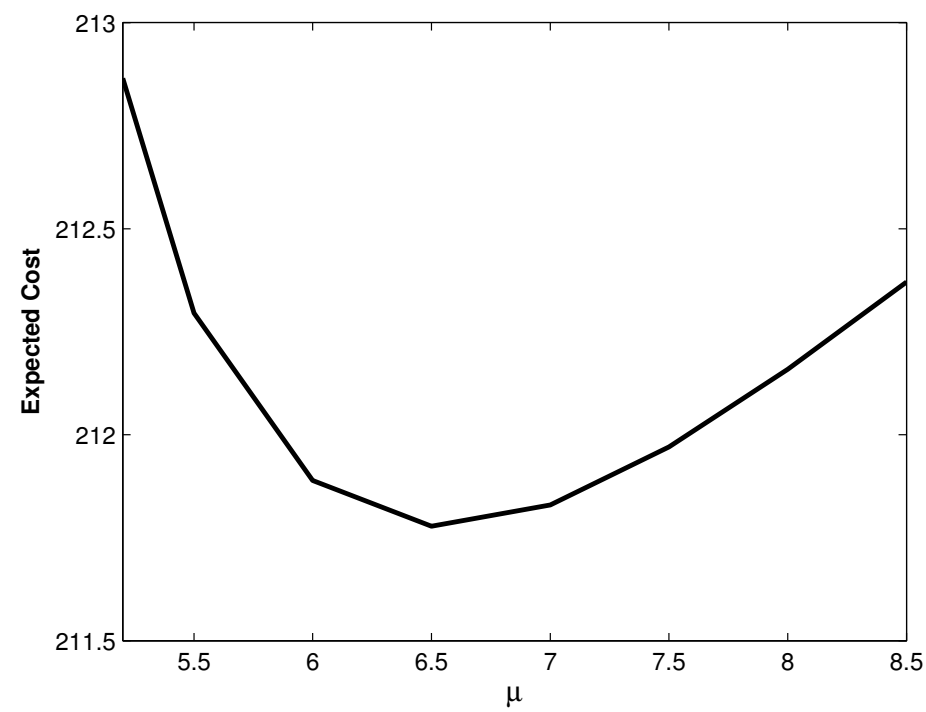

Figure 3: Impact of $\mu$ on expected cost.

Table 1: Search for optimum service rate during awake mode.

\begin{tabular}{|c|l|l|l|l|l|l|}
\hline No of iter. & $\mu_{0}$ & $\mu_{1}$ & $\mu_{2}$ & $\mu$ & $F(\mu)$ & Tolerance \\
\hline 1 & 6.0000 & 6.50000 & 7.00000 & 6.59144 & 211.77818 & .09144 \\
2 & 6.5000 & 6.59144 & 7.00000 & 6.54529 & 211.77754 & .04615 \\
3 & 6.5000 & 6.54529 & 6.59144 & 6.54528 & 211.77754 & .00001 \\
\hline
\end{tabular}

that $C_{1}=45, C_{2}=10, C_{3}=35$ and $C_{4}=5$. Figure 4 depicts the impact of service rate $\mu$ on the curve of the cost function. We use the quadratic fit search method [14] 
to find the optimum value of $\mu$, say $\mu^{*}$. According to the polynomial approximation theory, the unique optimum of the quadratic function agreeing with $f(x)$ at 3-point pattern $\left\{x_{0}, x_{1}, x_{2}\right\}$ occurs at

$$
x=\frac{1}{2} \frac{f\left(x_{0}\right)\left(x_{1}^{2}-x_{2}^{2}\right)+f\left(x_{1}\right)\left(x_{2}^{2}-x_{0}^{2}\right)+f\left(x_{2}\right)\left(x_{0}^{2}-x_{1}^{2}\right)}{f\left(x_{0}\right)\left(x_{1}-x_{2}\right)+f\left(x_{1}\right)\left(x_{2}-x_{0}\right)+f\left(x_{2}\right)\left(x_{0}-x_{1}\right)} .
$$

With the information of Figure 3, we choose the initial 3 point pattern $\mu_{0}=6.0, \mu_{1}=$ 6.5 and $\mu_{2}=7.0$. Table 1 indicates that the minimum expected operating cost per slot converges to the solution $\mu^{*}=6.54528$ with a optimal cost $F\left(\mu^{*}\right)=211.77754$. We assume the stopping tolerance limit as $10^{-4}$.

\section{Numerical results}

In this section, we present the numerical results and discuss the impact of system parameters on the performance indices. It establishes managerial insights on optimal decisions. Tables 2 and 3 present the optimum values of $\mu$ along with $L_{q}, L_{s}, P(B)$, $P(V), B R$ and $J S$ for various values of sleep rate $\phi$ and arrival rate $\lambda$, respectively. From Table 2, the decreasing trend is observed in $P(V), L_{q}, L_{s}, B R$ and $F\left(\mu^{*}\right)$ with the increase of sleep period $\phi$. That is, the number of data frames in BS buffer increases with extended sleep period. Furthermore, increasing trend is seen in $\mu^{*}$, $P(B)$ and $J S$ with the increase of sleep period $\phi$. It is observed from Table 3 that $P(B), L_{q}, L_{s}, B R, J S$ and $F\left(\mu^{*}\right)$ increase as the arrival rate $\lambda$ increases. But, decreasing trend is noted in $\mu^{*}$ and $P(V)$ with increase of arrival rate $\lambda$.

Figure 4 depicts the effect of $\rho$ on the average number of data frames in the

Table 2: System performance indices for various values of $\phi$.

\begin{tabular}{|c|c|l|l|}
\hline$\phi$ & $\phi=0.1$ & $\phi=0.3$ & $\phi=0.5$ \\
\hline$\mu^{*}$ & 5.500000 & 7.500000 & 9.500000 \\
$L_{q}$ & 7.278279 & 4.965582 & 3.701510 \\
$L_{s}$ & 7.468732 & 5.194701 & 3.907673 \\
$J S$ & 0.969650 & 1.511906 & 1.627788 \\
$B R$ & 1.452509 & 0.781608 & 0.541446 \\
$P(B)$ & 0.190453 & 0.229119 & 0.206164 \\
$P(V)$ & 0.809547 & 0.770881 & 0.793836 \\
$F\left(\mu^{*}\right)$ & 278.6682 & 195.7226 & 149.4758 \\
\hline
\end{tabular}

buffer $\left(L_{q}\right)$ for various balking functions. We assume the following four cases for the probability $b_{n}$ : (i) $b_{n}=1-n / N$, (ii) $b_{n}=1-n / N^{2}$, (iii) $b_{n}=1 /(n+1)$ and (iv) $b_{n}=1-e^{-n}$, where $n$ is the number of data frames and $N$ is the buffer size of the system. The probability $b_{n}$ is a monotonically decreasing function in the first two cases. The probability $b_{n}$ is an increasing function in the third and fourth cases. It is observed that the average number of data frames in the buffer $\left(L_{q}\right)$ decreases as traffic intensity $(\rho)$ increases. In case (ii), the average rate of balking of data frames 
Table 3: System performance indices for various values of $\lambda$.

\begin{tabular}{|c|c|l|l|}
\hline$\lambda$ & $\lambda=0.5$ & $\lambda=2.5$ & $\lambda=4.5$ \\
\hline$\mu^{*}$ & 9.500000 & 7.500000 & 6.500000 \\
$L_{q}$ & 1.432142 & 4.965582 & 6.007302 \\
$L_{s}$ & 1.477202 & 5.194701 & 6.443909 \\
$J S$ & 0.249020 & 1.511906 & 2.679488 \\
$B R$ & 0.071929 & 0.781608 & 1.662057 \\
$P(B)$ & 0.045060 & 0.229119 & 0.436607 \\
$P(V)$ & 0.954940 & 0.770881 & 0.563393 \\
$F\left(\mu^{*}\right)$ & 62.06171 & 195.7226 & 243.8471 \\
\hline
\end{tabular}

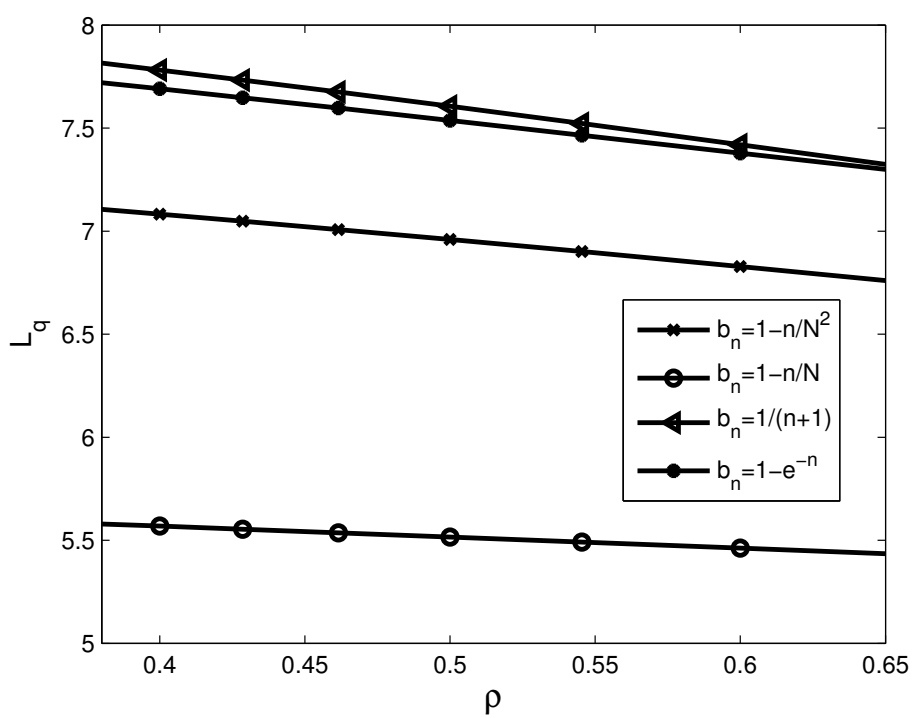

Figure 4: Effect of $\rho$ on $L_{q}$.

in the system is low.

Figure 5 shows the data frame loss $\left(P_{\text {loss }}\right)$ with the change of buffer size $N$ at various sleep rate $\phi$. The parameters are taken as $\lambda=3.0$ and $\mu=4.0$. We can see that data frame loss $\left(P_{\text {loss }}\right)$ ) decreases evidently with increasing value of buffer size $N$. It is also seen that with increase of sleep rate $\phi$, the data frame loss decreases monotonically. Figure 6 illustrates the dependence of balking rate $(B R)$ on the traffic intensity $(\rho)$. We observe that $B R$ decreases as the traffic intensity increases. For a fixed traffic intensity, the balking rate increases as the balking function decreases. Figure 7 shows the impact of traffic intensity $(\rho)$ on the $L_{q}$ and $J S$ for various sleep period $(\phi)$. It is seen that as $\rho$ increases the $L q$ decreases and the average rate of joining the system increases as it should be. For fixed $\rho$, as the sleep period $\phi$ increases 


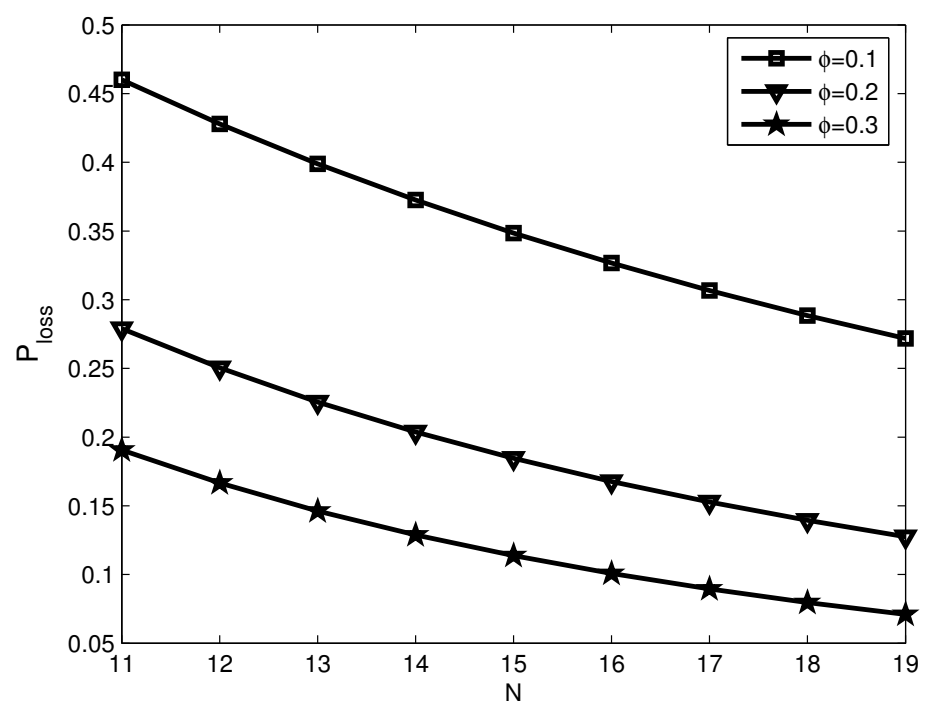

Figure 5: Impact of $N$ on $P_{\text {loss }}$.

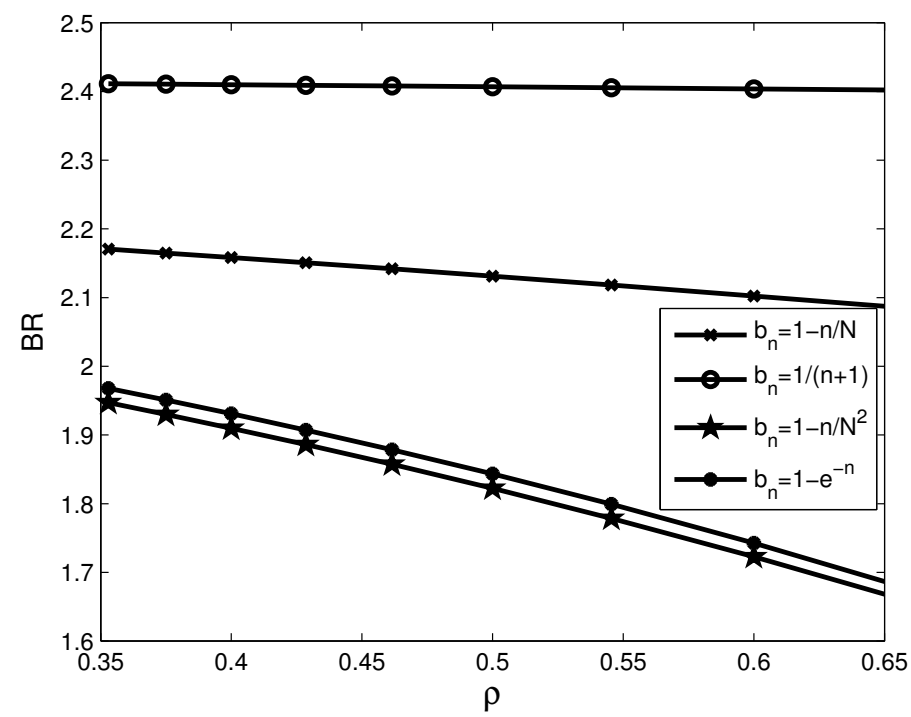

Figure 6: Effect of $\rho$ on $B R$.

the average number of data frames $L_{q}$ decreases and the average rate of joining the system increases. Hence, we can setup a traffic intensity $(\rho)$ and the sleep period $(\phi)$ 


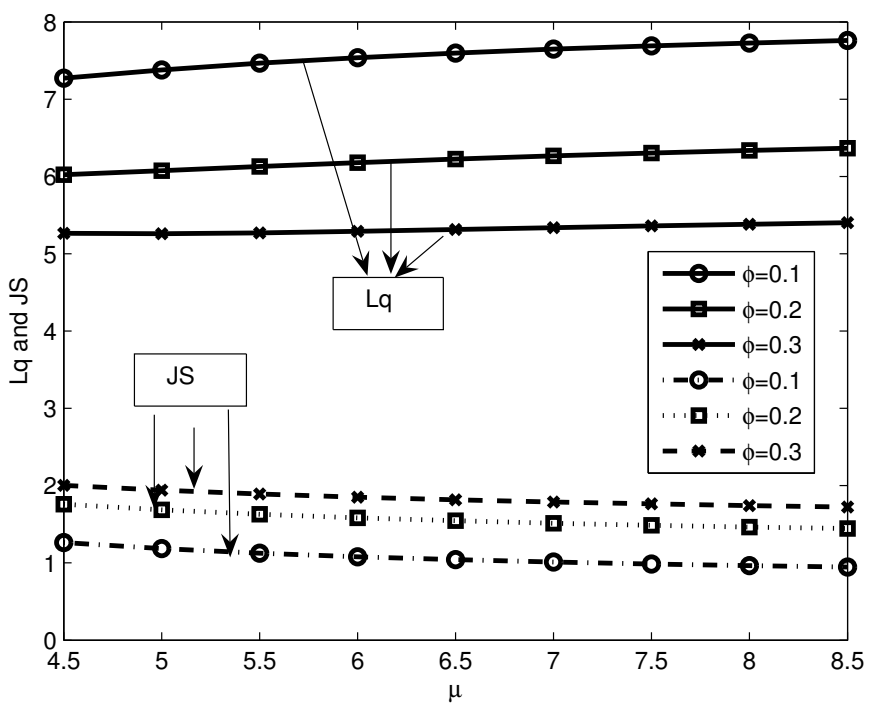

Figure 7: Impact of $\mu$ on the $L_{q}$ and $J S$.

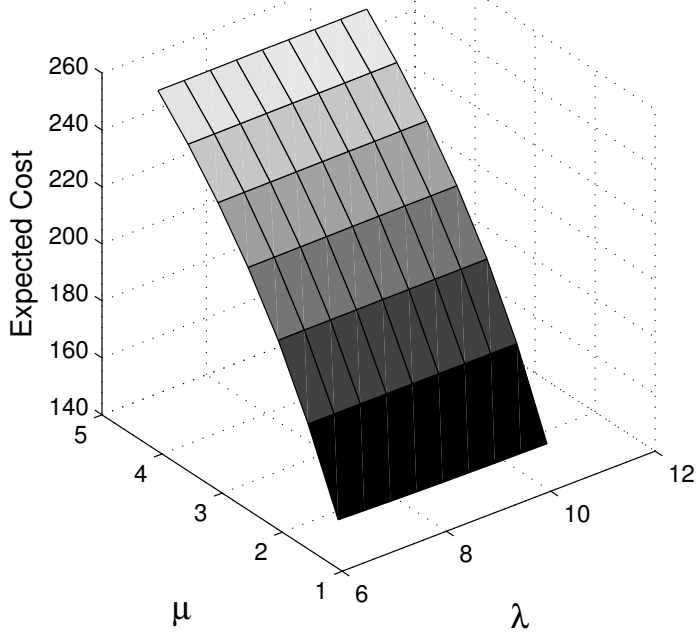

Figure 8: Effect of $\lambda$ and $\mu$ on expected cost

to minimize the average number of data frames and to maximize the average rate of joining the system. 


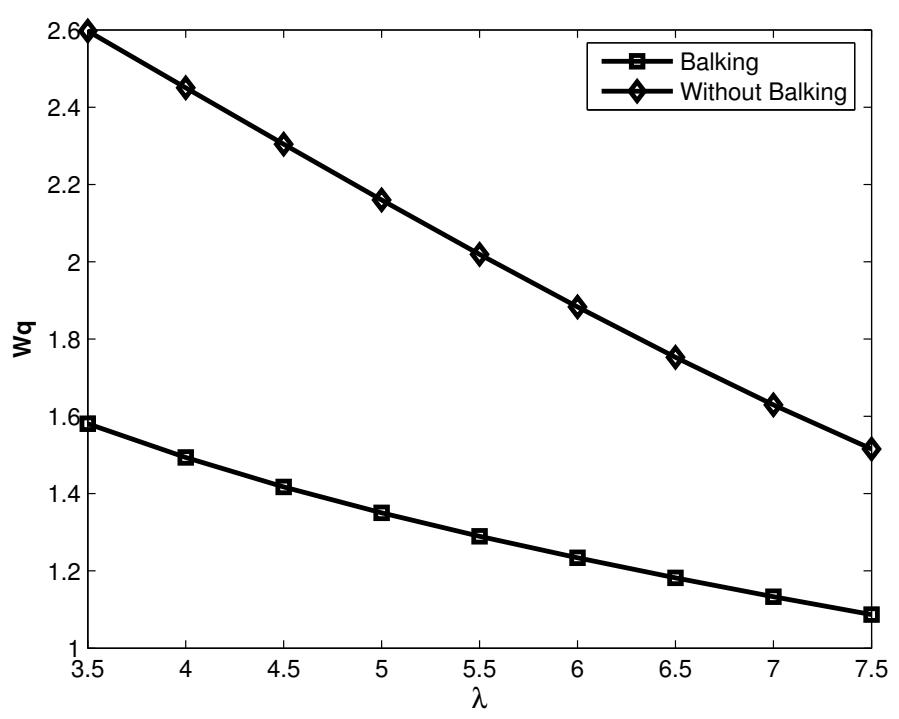

Figure 9: Effect of $\lambda$ and $W_{q}$

The impact of $\lambda$ and $\mu$ on cost function is shown in Figure 8. We observe that with the increase of service rate $\mu$, the cost function decreases. Further, with the increase of $\lambda$, the cost function increases. Thus, we can infer that the cost function depends on the choice of the service rates $\mu$ and data frames arrival rate $\lambda$ to a greater extent. Figure 9 plots the effect of $\lambda$ on the mean waiting time in the buffer $\left(W_{q}\right)$ for balking and without balking models. We can see that $W_{q}$ decreases evidently as the incoming traffic increases in both the models. It is easy to see that the balking model is better than the without balking model.

\section{Conclusions}

In this paper, we studied a real-time system that can handle impatient traffic in multiple sleep mode of the power saving class type II in the context of IEEE 802.16e standard. Under the proposed model, the incoming data frames may join or balk the buffer due to impatience with some probability. We presented an $M / M / 1 / N$ queueing model with balking and multiple sleep states in order to analyze the performance of the power saving class type II for delay sensitive traffic. We also developed a cost function to determine the optimal service rate that minimizes the total expected cost. Various performance measures such as the average number of data frames in the system, the average number of data frames in the buffer, the average waiting time of data frames, data frame loss due to buffer size limitation, and the rate of abandonment due to impatience are evaluated. We observed from the numerical results that our proposed 
multiple sleep state with balking can save more energy with the same throughput and delay. The proposed model provides a tradeoff between the average abandon rate and the power consumption and it may be useful for the design of the power-saving systems under real-time traffics.

\section{References}

[1] Baek S., Choi B.D., Performance of an efficient sleep mode operation for IEEE 802.16m, Journal of Industrial and Management Optimization, 7, 3, 2011, 623639 .

[2] Chen C.Y., Hsu C.H., Feng K.T., Performance analysis and comparison of sleep mode operation for IEEE $802.16 \mathrm{~m}$ advanced broadband wireless networks, Proceedings of the IEEE 21st International Symposium on Personal Indoor and Mobile Radio Communications, 2010, 1425-1430.

[3] Han K., Choi S., Performance Analysis of Sleep Mode Operation in IEEE 802.16e Mobile BroadBand Wireless Access Systems, The 12th Asia-Pacific Conference on Communications, 2006, 1141-1145.

[4] Hwang E., Kim K.J., Son J.J., Choi B.D., The power saving mechanism with binary exponential traffic indications in the IEEE $802.16 \mathrm{e} / \mathrm{m}$, Queueing Systems, 62, 3, 2009, 197-227.

[5] Hwang E., Kim K.J., Son J.J., Choi B.D., The power-saving mechanism with periodic traffic indications in the IEEE $802.16 \mathrm{e} / \mathrm{m}$, IEEE Transactions on Vehicular Technology, 59, 1, 2010, 319-334.

[6] IEEE 802.16 Working Group, IEEE Standard for Local and Metropolitan Area Networks. Part 16: Air Interface for Fixed and Mobile Broadband Wireless Access Systems. Amendment 3: Management Plane Procedures and Services, IEEE Standard 802, 2006.

[7] Jang J., Han K., Choi S., Adaptive power saving strategies for IEEE 802.16 e mobile broadband wireless access, In Communications, APCC'06. Asia-Pacific Conference, 2006, 1-5.

[8] Javed U., Suchara M., He J., Rexford J., Multipath protocol for delay-sensitive traffic, In Communication Systems and Networks and Workshops, COMSNETS, 2009, 1-8.

[9] Jin S., Yue W., Performance analysis and evaluation for power saving class type III in IEEE 802.16e network, Journal of Industrial and Management Optimization, 6, 3, 2010, 691-708.

[10] Jin S., Chen X., Qiao D., Choi S., Adaptive sleep mode management in IEEE 802.16m wireless metropolitan area networks, Computer Networks, 55, 16, 2011, 3774-3783. 
[11] Jones C.E., Sivalingam K.M., Agrawal P., Chen J.C., A survey of energy efficient network protocols for wireless networks, International Journal of wireless networks, 7, 4, 2001, 343-358.

[12] Lee J., Cho D., An optimal power-saving class II for VoIP traffic and its performance evaluations in IEEE 802.16 e, Computer Communications, 31, 14, 2008, 3204-3208.

[13] Lee K., Mun Y., Enhanced power saving mechanism for Type I and Type II power saving classes in IEEE 802.16e, IEICE Transactions on Communications, E94-B, 9, 2011, 2642-2645.

[14] Rardin R.L., Optimization in Operations Research, Prentice Hall, 1999.

[15] Seo J.B., Lee S.Q., Park N.H., Lee H.W., Cho C.H., Performance Analysis of Sleep Mode Operation in IEEE 802.16e, Proc. IEEE VTC, 2, 2004, 1169-1173.

[16] Wongthavarawat K., Ganz A., Packet scheduling for QoS support in IEEE 802.16 broadband wireless access systems, International Journal of Communication Systems, 16, 1, 2003, 81-96.

[17] Yue D., Zhang Y., Yue W., Optimal performance analysis of an $M / M / 1 / N$ queue system with balking, reneging and server vacation, International Journal of Pure and Applied Mathematics, 28, 1, 2008, 101-115.

[18] Zhang Y., Performance modeling of energy management mechanism in IEEE 802.16e mobile WiMAX, Wireless Communications and Networking Conference, 2007, 3205-3209.

Received 31.01.2014, accepted 27.10.2014 